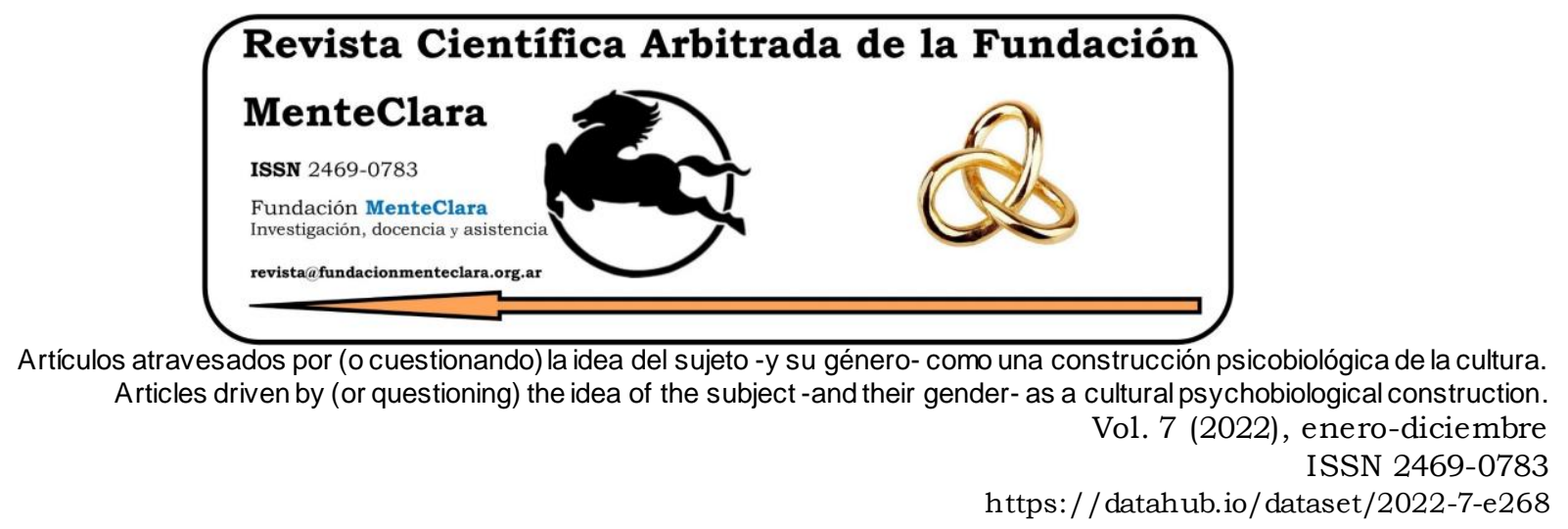

\title{
LA NEURODIDÁCTICA EN LOS PROCESOS DE ENSEÑANZA Y APRENDIZAJE ¿UN NUEVO PARADIGMA EN EDUCACIÓN?
}

\author{
NEURODIDACTIC IN THE TEACHING AND LEARNING PROCESSES, A NEW \\ PARADIGM IN EDUCATION?
}

Wilmer Casasola Rivera wcasasola@itcr.ac.cr

Tecnológico de Costa Rica, Escuela de Ciencias Sociales, Costa Rica

Cómo citar este artículo / Citation: Casasola Rivera, W. (2022). La neurodidáctica en los proce sos de enseñanza y aprendizaje ¿un nuevo paradigma en educación? Revista Cientifica Arbitrada de la Fundación MenteClara, Vol. 7 (268). DOI:

https: / / doi.org/ 10.32351/rca.v7.268

Copyright: (C) 2022 RCAFMC. Este artículo de acceso abierto es distribuido bajo los términos de la licencia Creative Commons Attribution 4.0 International License (CC BY 4.0). Recibido: 27/01/2022. Aceptado: 04/02/2022 Publicación online: 11/02/2022

Conflicto de intereses: Ninguno que declarar.

\section{Resumen}

Este artículo es una reelaboración completa de una ponencia presentada en el año 2015, en el V Congreso Internacional de Administración Educativa CIAE, realizado en San José, Costa Rica, con el título "Neurodidáctica: hacia un nuevo paradigma educativo". Para la elaboración de este artículo se introducen algunas fuentes y replanteamiento de ideas. La neurodidáctica es un enfoque pedagógico producto de investigaciones en el campo de las neurociencias. El problema y objetivo que asume este artículo es discutir y analizar si se puede demarcar la neurodidáctica como paradigma en educación y, a la vez, reflexionar sobre la importancia de la neurodidáctica en los procesos de enseñanza y aprendizaje. Se parte de un enfoque cualitativo y una técnica de investigación documental. La revisión de la literatura es no sistemática en la medida en que la selección de fuentes queda sujeta a los criterios del autor. 


\begin{abstract}
This article is a complete reworking of a paper presented in 2015, at the V International Conference on Education Administration CIAE, held in San José, Costa Rica, with the title "Neurodidactics: towards a new educational paradigm". For the elaboration of this article some sources and rethinking of ideas are introduced. Neurodidactics is a pedagogical approach resulting from research in the field of neuroscience. The problem and objective that is assumed in this article is to discuss and analyze whether neurodidactics can be referred a paradigm in education and, at the same time, reflect on the importance of neurodidactics in the teaching and learning processes. The approach is qualitative and the research technique is documentary. The review of the literature is non-systematic. The selection of sources is subject to the author's discretion.
\end{abstract}

Palabras Claves: Neurodidáctica; aprendizaje; enseñanza; paradigma; neurociencia; neurobiología; cerebro; pe dagogía; educación

Keyw ords: Neurodidactics, learning, teaching, paradigm, neuroscience, neurobiology, brain, pedagogy, education 


\section{Introducción}

Este artículo parte de algunas ideas de una ponencia presentada en el año 2015, en el $V$ Congreso Internacional de Administración de la Educación CIAE, celebrada en San José, Costa Rica, con el título "Neurodidáctica: hacia un nuevo paradigma educativo". Aquí se hace una reelaboración completa y se introducen algunas fuentes y el replanteamiento de ideas. La ponencia se presentó originalmente en diapositivas, como conversatorio, y nunca hubo intención de publicar su contenido.

Los modelos didácticos son herramientas que permiten a docentes mejorar su experiencia pedagógica. El empleo de un modelo didáctico tiene que ver con la funcionalidad del mismo modelo en la práctica docente.

Cuando un modelo didáctico o pedagógico ofrece ciertas dificultades para explicar la dinámica de la experiencia de aprendizaje es necesario revisar el modelo y considerar la necesidad de incorporar un nuevo modelo didáctico o pedagógico que contribuye con un mejor proceso de aprendizaje. Sin embargo, no todo modelo didáctico se puede demarcar como paradigma.

El tema de este artículo de reflexión se enfoca en discutir si la neurodidáctica se puede considerar un paradigma en educación y reflexiona sobre la importancia de la neurodidáctica en el proceso de enseñanza y aprendizaje. De forma puntual, el problema que intenta discutir plantea la siguiente pregunta: ¿se puede demarcar la neurodidáctica como paradigma en educación? Como objetivo general persigue analizar si la neurodidáctica se puede considerar un paradigma 
y a la vez, reflexionar sobre la importancia de la neurodidáctica en los procesos de enseñanza y aprendizaje.

El artículo se aborda desde un enfoque cualitativo a través de una técnica de investigación documental. Revisa literatura de forma no sistemática, en la medida en que las fuentes seleccionadas son aquellas que fundamentan aspectos generales que el autor quiere desarrollar. Parte de una descripción bibliográfica y no tiene la finalidad, por la naturaleza del trabajo, discutir resultados, sino ofrecer un panorama general del tema. La perspectiva desde la cual se aborda el trabajo obedece a un análisis de contenido.

El trabajo aporta una serie de análisis e interpretaciones sobre la neurodidáctica. En este sentido, su contenido enriquece la discusión sobre el tema en el campo de la educación.

La neurodidáctica no puede asumirse como un paradigma. Esta afirmación se formula de acuerdo con los planteamientos epistemológicos de Thomas Kuhn en su obra The structure of scientificrevolutions, de1962 (Kuhn, 2004).

Los paradigmas surgen al interior de una comunidad científica y sirven como modelo para que las comunidades científicas desarrollen toda su actividad investigativa. Se trata de un conjunto de planteamientos teóricos, normas, valoraciones y procedimientos que comparten los miembros de una comunidad científica en particular, que orientan la práctica científica en la medida que permite o posibilita el conjunto de procedimientos metodológicos para producir y reproducir un determinado conocimiento científico. 
Dado que el tema no se ha abordado con esta delimitación, no se cuenta con antecedentes. No obstante, el trabajo no persigue esta finalidad. El propósito del artículo es reflexionar sobre la importancia de la neurodidáctica y discutir si se le puede considerar como paradigma. Para este fin, intenta exponer algunas ideas contenidas en la literatura académica.

\section{Breve aproximación a la noción de paradigma}

Para determinar si la neurodidáctica se puede asumir como un paradigma, es importante realizar algunas acotaciones sobre este concepto.

El concepto de paradigma se utilizó originalmente en gramática, retórica, lingüística y posteriormente en psicología. El significado actual que conocemos de paradigma relacionado con la práctica científica se debe al físico y filósofo de la ciencia Thomas Kuhn (1922-1996). El

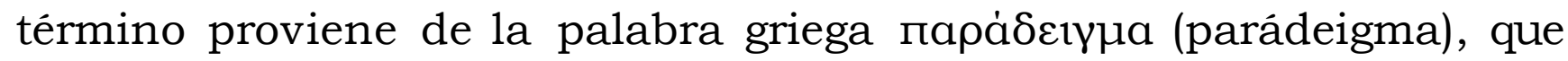
significa simplemente modelo. Una forma sencilla de comprender la noción de paradigma es como modelo explicativo de la realidad (Casasola Rivera, 2010)

Un paradigma surge al interior de estas comunidades y es la base teórica para desarrollar cierta actividad investigativa. Se puede decir que un paradigma es una especie de modelo epistemológico. Se compone de normas, valoraciones y procedimientos que sirven para orientar la práctica científica en la medida en que permite o posibilita la selección de las técnicas y procedimientos conducentes a un quehacer científico objetivo y riguroso. Kuhn señala, en la Posdata de 1969, que un paradigma es aquello que comparten los miembros de una comunidad cientifica en particular (Kuhn, 2004) (Casasola Rivera, 2010) 
Los paradigmas están asociados a las revoluciones científicas. No siempre las teorias logran explicar satisfactoriamente algún aspecto de la realidad o fenómeno. Las anomalías están presentes en muchas teorias científicas.

Si un planteamiento nuevo permite explicar, de manera más completa, un fenómeno de la realidad y ofrece elementos para reemplazar las explicaciones anteriores, o bien, ampliarlas, se dice que es un nuevo paradigma. Un nuevo paradigma podría provocar, al generalizarse, una revolución científica.

La ciencia normal es el modelo explicativo que sustentamos, lo que nos permite tener alguna certeza sobre la realidad y el comportamiento de los fenómenos u objetos de estudio. Es la ciencia en la que creemos. Pero cuando esta ciencia normal vigente, cuando este paradigma o modelo científico aceptado universalmente no puede explicar ciertas anomalias, se empieza a gestar la revolución científica. Surge la necesidad de un nuevo paradigma científico. La ciencia sufre un fenómeno espiral. La ciencia normal se basa en un paradigma vigente. A partir de este paradigma, la comunidad cientifica explica, desde su disciplina, la realidad o fenómeno de estudio. Sin embargo, cuando este modelo no logra explicar enteramente el fenómeno de la realidad que se estudia, surgen las paradojas, las anomalías, las crisis al interior del paradigma o del modelo teórico (Casasola Rivera, 2010)

Esta dificultad que experimenta una teoria para explicar la realidad, provoca la necesidad de buscar otros modelos explicativos de la realidad, otras teorias. Si una teoría logra explicar satisfactoriamente algún segmento la realidad, estamos ante un nuevo paradigma.

Los paradigmas operan durante cierto tiempo como modelos explicativos de la realidad, se aceptan y utilizan por la comunidad 
científica en su quehacerinvestigativo rutinario (Casasola Rivera, 2010). Esto sería ciencia normal, la ciencia que hoy aceptamos como verdadera. Esta tranquilidad dogmática se mantiene hasta que empezamos a advertir que algo no logra explicarse adecuadamente.

\section{Acercarse a la neurobiologia del aprendizaje}

La neurodidáctica tiene una fuerte fundamentación en hallazgos científicos en el campo de la neurobiología, especialmente en lo que tiene que ver con el funcionamiento del cerebro y el aprendizaje.

La neurobiología estudia las células del sistema nervioso en toda su amplitud con el fin de comprender cómo el cerebro procesa la información que recibe, dado que es la base para el comportamiento humano (Ugaz Cayao, Fernández Cosavalente, Ugaz Cachay, Vásquez Vásquez, \& Quiroz Aldave, 2019). Estos estudios tienen gran relevancia para comprender procesos básicos del comportamiento en los entornos educativos. Por esta razón, se habla de una neurobiología del aprendizaje.

Hemos asumido la enseñanza, no pocas veces, como un ejercicio empírico y magistral. El modelo magistral persiste en los ambientes educativos. En el contexto universitario la clase magistral es el recurso didáctico por excelencia. ¿Responde el modelo magistral de enseñanza a las necesidades de aprendizaje de los estudiantes en el salón de clases?

Los avances en neurobiología y neurocognición se consideran fundamentales para comprender de la dinámica del cerebro y la forma como aprende. Pese a estos avances, existe cierta persistencia de apegarse a modelos tradicionales de enseñanza y desatender cómo aprende el cerebro y qué factores facilitan el aprendizaje.

El cerebro humano contiene miles de millones de neuronas en continuo contacto sináptico entre células y de forma muy variable. La 
sinapsis es el medio por el cual existe un contacto funcional entre una neurona y otra. Es el mecanismo por el cual existe la transferencia de información entre células: las células presinápticas y las células postsinápticas. Hebb había demostrado la importancia de la actividad presináptica y postsináptica de las neuronas, porque fortalece la interconexión entre ellas. La activación y asociación simultanea de células, la comunicación neuronal, se asocia a los mecanismos fisiológicos del aprendizaje y la memoria (Uchitel, 2007) (Adrover, Muñoz, Sánchez, \& Miranda, 2017). De ahí que existe toda una discusión en el ámbito de la neuroeducación sobre la forma como se establece una mejor estimulación para que estos mecanismos fisiológicos se potencian a través de diferentes estrategias didácticas.

Una pregunta siempre vigente y abierta a investigación es ¿Qué es el aprendizaje? En el contexto neuropsicológico se comprende como el proceso cerebral que implica análisis, tratamiento, almacenamiento y recuperación de la información que recibimos del mundo exterior a través de diferentes medios. En general, para las neurociencias, aprendizaje es el proceso mediante el cual las experiencias modifican nuestro sistema nervioso y, desde este, nuestra conducta (Pérez, 2014).

Una característica neurobiológica del cerebro humano es estar preparado para adaptarse de acuerdo con el aprendizaje del entorno. La diversidady complejidad de los estímulos del mundo exterior determinan la complejidad con la que se conectan las neuronas y se produce el aprendizaje. En el contexto educativo significa que debe existir una adecuada estimulación externa que posibilite el proceso lógico de aprendizaje a través de la formación de redes o conexiones neuronales. Por ejemplo, diseñar estrategias para potenciar el proceso atencional. La atención nos permite discriminar entre estímulos relevantes e 
irrelevantes. Por esta razón es fundamental conocer el mecanismo de los diferentes tipos de memoria y la forma como trabajan.

Investigaciones recientes señalan que la atención sostenida tiene un sustrato fisiológico, regulado por el sistema nervioso autónomo, aunque el componente psicológico es fundamental para este tipo de regulación. El potencial para aprender está condicionado por los conocimientos previos, las emociones, las metas y especialmente por las actividades que se propicien en el salón de clase, porque son los mecanismos que crean y sustentan redes neuronales. A mayor cantidad de conexiones de neuronas en las áreas cerebrales, mayor será la comunicación eficaz entre ellas. De esta forma, la atención sostenida en los procesos de enseñanza y aprendizaje alcanzará una mejor eficacia. La función del sistema nervioso autónomo, a través de sus dos sistemas, el simpático y el parasimpático, consiste el trabajo regulatorio que realiza: a la vez que un sistema prepara para la acción ante potenciales amenazas, acelerando la frecuencia cardiaca, el otro sistema mantiene al organismo un estado corporal de reposo (Rodríguez de Ávila, 2020). Las estrategias didácticas deben potenciar una sana regulación de estos sistemas. La pedagogía tradicional se ha fundamentado en el temor: los exámenes memorísticos son una clara representación de esta pedagogía del miedo.

La enseñanza requiere de estrategias eficaces que garanticen el aprendizaje. Según el tipo de enseñanza, se puede potenciar en estudiantes habilidades metacognitivas que les permitan desarrollar adecuadamente sus capacidades intelectuales para procesar la información y aplicarla en la solución de problemas en el entorno educativo o en la interacción social.

El aprendizaje se procesa en el cerebro. La memoria es un elemento fundamental para el aprendizaje. La memoria y el aprendizaje se 
relacionan de forma complementaria. El aprendizaje produce una serie de cambios en el cerebro que permiten la consolidación de la memoria en sus distintas variantes, hasta alcanzar su posterior almacenamiento a largo plazo. Comprender el funcionamiento del cerebro en los procesos de aprendizaje nos permite una mejor intervención en la enseñanza. El reto que tenemos como educadores e investigadores es determinar las etapas y los estímulos adecuados que posibilitan un aprendizaje significativo.

El aprendizaje depende de la memoria para su consolidación y funcionalidad. Se podría afirmar que es una relación dialéctica: la memoria es el producto del aprendizaje y el aprendizaje permite que la memoria se consolide. En el proceso de aprendizaje, las asociaciones que se generan modifican las conexiones sinápticas de los circuitos cerebrales involucrados.

La pregunta que se hacen los investigadores es ¿cómo aprende el cerebro? El concepto que logra explicar cómo nuestro cerebro construye su aprendizaje se denomina plasticidad cerebral o neuroplasticidad.

El concepto neuroplasticidad cerebral hace referencia a la capacidad del cerebro para modificar su conducta a través del aprendizaje activo. Esta destreza o plasticidad para reorganizar sus funciones y generar nuevas conexiones, le permite al cerebro aprender nuevas cosas a cualquier edad. En cada etapa del desarrollo del ser humano, la plasticidad neuronal experimenta distintos cambios en su proceso de aprendizaje. Los cambios que experimenta el cerebro y su capacidad de aprendizaje se relacionan con las conexiones celulares y con la velocidad de comunicación de las células neuronales (Landivar, 2012).

Cada etapa de desarrollo cuenta con ventajas y desventajas en el proceso de aprendizaje. No obstante, el cerebro humano nunca deja de 
aprender. La neurogénesis, o nacimiento de nuevas neuronas, rechaza la creencia de una interrupción del aprendizaje al alcanzar cierta edad. Por el contrario, hoy contamos con investigaciones que demuestran que no existe una edad límite para el desarrollo cerebral. A cualquier edad seguimos creando nuevas conexiones neuronales.

La plasticidad neuronal o neuroplasticidad es una característica propia del cerebro. Como educadores, debemos diseñar estrategias didácticas enfocadas a estimular esta plasticidad que se reflejarán en una mejor experiencia de aprendizaje de acuerdo con el desarrollo neurobiológico de niños, adolescentes y adultos. La neuroplasticidad se puede modificar en cualquier etapa del desarrollo humano, es decir, tanto en la infancia y adolescencia, como en la edad adulta. De ahí que los factores ambientales y sociales son fundamentales para su adecuada estimulación, porque permiten establecer conexiones sinápticas que favorecen el aprendizaje.

El cerebro ha evolucionado con una plasticidad que le permite reorganizarse y aprender continuamente. El cerebro aprende a través de diferentes maneras y con una variedad de recursos. Enfocarse en estudiar cómo funciona y aprende el cerebro puede aportar un conocimiento valioso a la educación en todos sus niveles. Esto nos obliga a reconsiderar los estilos de enseñanza unilaterales y monótonos que no permiten aprovechar la capacidad que tiene el cerebro para aprender, y que afecta significativamente el deseo natural que todos tienen por aprender.

La educación como ciencia pueden tener múltiples objetivos. Uno de estos objetivos puede ser, de acuerdo con Pérez (2014), desarrollar especialistas en aprendizaje, que el objetivo que se busca con el nuevo conocimiento que adquirimos del cerebro. Para lograr este cambio y 
abandonar la verticalidad pedagógica se debe saber cómo funcionan las estructuras cerebrales y la fisiología básica del aprendizaje para correlacionarlos con las conductas y hábitos que más favorezcan el aprendizaje de los estudiantes.

\section{III. ¿Se puede asumir la neurodidáctica como un paradigma en educación?}

Asumir un cuerpo teórico de conocimiento como paradigma, significa asumir que este conocimiento logra explicar, satisfactoriamente, un fenómeno de la realidad.

Un nuevo paradigma es un modelo teórico que permite explicar de manera más completa un fenómeno de la realidad. Este nuevo cuerpo de conocimiento permite reemplazar o ampliar las explicaciones anteriores, subsanar las anomalias. En este contexto, cabe pensar si la didáctica general o las didácticas especiales no logran explicar o abordar cierto fenómeno en el campo de la educación, al punto de considerar la neurodidáctica como un nuevo paradigma, una revolución científica educativa.

En la práctica educativa podríamos ubicar dos perfiles básicos extremistas: los neurofílicos y los neurofóbicos. Un neurofílico es el educador que pretende reducir toda la educación a la neurociencia y sus hallazgos. Un neurofóbico es el educador tradicional que niega toda posibilidad de una neurociencia educativa.

Existe, no obstante, para parodiar a Aristóteles, un justo medio o una tercera vía: la neuroinvestigación. Aquí podriamos ubicar a las personas que formalmente se han especializado en neuroeducación y cuentan con las herramientas teóricas, científicas y metodológicas para aplicar y gestionar investigaciones que unifiquen la neurociencia con la educación. 
O bien, lo ideal una sociedad del conocimiento complejo: los equipos de investigación interdisciplinarios.

Ahora bien, ante cualquier posición que adoptemos, ya sea defender o rechazar la neuroeducación y sus derivaciones, como la neurodidáctica, se tiene que contar con una sólida formación en algún campo neurocientífico o neuroeducativo. Cabe pensar, sin embargo, que muchos neurofílicos y neurofóbicos no cuenten formalmente con formación en alguna neurociencia o desconozcan el marco metodológico y epistemológico de la neuroeducación. Aquí la discusión se complica.

Por otra parte, tenemos que considerar una variable importante que puede influir significativamente en las posturas de los neurofílicos y los neurofóbicos: la usencia de formación en educación. Este fenómeno es más frecuente en los profesores universitarios: son profesionales en alguna área, pero no necesariamente cuentan con formación en docencia.

Muchos profesionales involucrados en educación (sin formación docente) rechazan de forma despectiva los alcances de la neuroeducación. Posiblemente porque aún no conocen, si quiera, los principios básicos de la educación como disciplina científica, y eso los impulsa a rechazar lo que no conocen. También, muchos docentes están aferrados a modelos pedagógicos basados en su propia práctica educativa, posiblemente replican, en el salón de clases, el modelo pedagógico bajo el cual fueron formados. En el otro extremo, tenemos los profesionales que creen que se puede hacer tabula rasa en nombre de la neuroeducación, y tirar por la borda todo el bagaje teórico que se ha producido en las ciencias de la educación a través de los años. Además, son profesionales que consideran que se puede generalizar cualquier avance en el campo de las neurociencias. Esta última postura es muy característica de los neuromitos. Ambos enfoques están errados. 
En esto tenemos que operar con un principio de objetividad. Hay posturas parciales, que provienen de algunas ciencias, que opinan sobre la actividad profesional de la educación sin evidenciar alguna formación educativa. La postura del médico Tomás Ortiz es un claro ejemplo de esto. Afirma que la educación no es una disciplina que tiene por objetivo la investigación y que por esta razón tiene una limitada capacidad explicativa o predictiva, desde un punto de vista científico (Ortiz, 2015). Esta postura es reduccionista y cientificista. Intenta descalificar una ciencia porque no opera bajo los esquemas metodológicos de otra ciencia. Esta afirmación demuestra cierto grado de desconocimiento epistemológico, porque parte de una demarcación científica generalizada, sin tomar en cuenta que, apelar hoy a un modelo único de ciencia, es un serio error epistemológico. Sorprende que, luego de tanta cultura epistémica, algunos sigan aferrados a esquemas positivistas. Otro médico, Francisco Mora, considera, por el contrario, que la neuroeducación es una nueva visión de la enseñanza basada en el cerebro, aunque estos conocimientos no se puedan aplicar de forma sistemática en los centros de enseñanza (Mora, 2016 ).

No solo existe investigación educativa y propuestas teóricas que mejoran los procesos de enseñanza y aprendizaje, sino aplicación constante de investigaciones científicas en otros campos. Desde hace muchos años ponemos en práctica en el salón de clases, descubrimientos científicos basados en el estudio del cerebro. La psicología del aprendizaje es una muestra de esto. Hoy solo ampliamos los criterios con datos que provienen de nuevas técnicas de estudio del cerebro humano.

La neurociencia estudia las funciones específicas del tejido nervioso y su relación con la mente y la conducta. La comprensión del funcionamiento del cerebro nos permitiría comprender de mejor manera el proceso de enseñanza y aprendizaje. 
La neurodidáctica es un enfoque pedagógico que se basa en investigaciones que provienen de diferentes disciplinas, y que tiene una fuerte inspiración en las neurociencias. La neurociencia estudia el sistema nervioso desde un punto de vista multidisciplinario. Son muchas las disciplinas que conforman lo que se conoce como neurociencias, incluyendo a la neuropsicología.

Las neurociencias buscan descifrar la fisiologia cerebral de la cognición y disponer de modelos funcionales que puedan explicar el funcionamiento cognitivo en la normalidad y que puedan predecir qué ocurrirá en una situación de daño cerebral (Portellano \& García, 2014). En este sentido, es un conjunto de saberes que permite comprender de forma más rigurosa el funcionamiento del cerebro. Por ejemplo, el encéfalo se relaciona con la producción y ejecución de acciones vinculadas directamente con la conducta, el aprendizaje y la memoria en sus diferentes modalidades.

Las neurociencias son un conjunto de disciplinas que permiten un conocimiento más amplio del cerebro: qué lo afecta, cómo funciona, cómo aprende, entre otros. En educación tenemos que aproximarnos a las investigaciones neurocientíficas sobre el funcionamiento del cerebro y evaluar de forma rigurosa si estos resultados se pueden aplicar al campo de la educación y de qué forma. Esto implica realizar investigaciones con diseños exclusivos para plantear y verificar hipótesis de trabajo. Esto nos podría dar insumos para rediseñar propuestas didácticas y mejorar la experiencia de aprendizaje.

Dese hace muchos años contamos con modelos didácticos para la enseñanza y el aprendizaje. Algunos datos actuales, sobre cómo funciona mejor la atención o la memoria, por citar solo dos ejemplos, pueden enriquecer nuestro modelo didáctico, al incorporar estos 
descubrimientos y ajustarlos a los modelos pedagógicos. Pero debemos orientarnos bajo un principio de objetividad: no toda investigación neurocientífica se puede generalizar al campo de la educación, como tampoco, no todo el conocimiento neurocientífico se puede ignorar en educación. Si ignoramos estos avances, nos sumergimos en una cultura de la desactualización profesional. Implementar estrategias didácticas con apoyo en los nuevos datos de la neurociencia, puede contribuir positivamente con una mejor enseñanza y aprendizaje.

Ahora bien, la pregunta central de este artículo plantea: ¿Se puede asumir la neurodidáctica como un nuevo paradigma en educación? Una respuesta preliminar señala que no. Sin embargo, se puede asumir la respuesta a esta pregunta como la apertura al debate académico.

El neologismo neurodidáctica rescata el trabajo de muchos teóricos de la cognición educativa, como Piaget y Vygotsky, por citar solo dos figuras. Pero hoy, gracias a nuevas investigaciones e instrumentos tecnológicos contamos con mayor evidencia sobre el funcionamiento cerebral. La aplicación de estos descubrimientos, bajo una fundamentación epistemológica consistente, puede proporcionarnos un modelo didáctico para trabajar la enseñanza y lograr un mejor aprendizaje.

La neurodidáctica se fundamenta en un conjunto de disciplinas. Se puede decir que la neurodidáctica es parte de los procesos de investigación e innovación pedagógica.

La neurodidáctica es la aplicación de conocimientos sobre el funcionamiento del cerebro y de cómo intervienen los procesos neurobiológicos en el aprendizaje, con el fin de optimizar su funcionamiento (Forés Miravalles \& Ligioiz Vázquez, 2015). La neurodidáctica busca unificar descubrimientos acerca del funcionamiento de cerebro y ponerlo al servicio del aprendizaje. 
En el contexto de la construcción y aplicación de estrategias neurodidácticas, la neuropsicología ocupa un lugar fundamental, especialmente la neuropsicología educativa, dada su capacidad de intervención. La neuropsicología estudia y trata las funciones mentales superiores en sus relaciones con las estructuras cerebrales. Por eso es importante conocer qué tipo de aplicación tiene la neuropsicología en el desarrollo del aprendizaje escolar del niño

La neuropsicología nos enseña que el aprendizaje tiene que ir en consonancia con el desarrollo y maduración del cerebro y sus funciones. A esto se le da el nombre de neuroplasticidad. En función del desarrollo y evolución del cerebro, la neurodidáctica podría trabajar con los estímulos adecuados para conseguir un aprendizaje óptimo, promoviendo un cambio en el desarrollo de las capacidades cognitivas de las personas.

Algunos consideran que la neurodidáctica es una ciencia, una mezcla entre la didáctica y la neurociencia. O bien, una rama de la pedagogía que tiene como fin diseñar estrategias más eficientes para lograr un mejor aprendizaje. También, de forma más modesta, otros consideran la neurodidáctica como una aplicación de conocimientos sobre el funcionamiento del cerebro y el papel que tienen los procesos neurobiológicos en el aprendizaje (Pherez, Vargas, \& Jerez, 2018).

El objetivo de la neurodidáctica es ofrecer herramientas más efectivas en la optimización del aprendizaje. Se enfoca en los procesos neurobiológicos y neurocognitivos que condicionan la conducta y el aprendizaje. El aprendizaje cambia la estructura del cerebro. La conducta humana cambia. La estructura del cerebro determina la forma del comportamiento humano en sociedad. 
No obstante, la neurodidáctica no parece reunir los requisitos de un paradigma, según la epistemologia kuhniana. Para empezar, se tiene que tomar en cuenta que existen dos modalidades de la didáctica: la general y la especial. Dentro de las didácticas especiales, contamos con una gran variabilidad de posturas didácticas. No se puede generalizar neurodidáctica con didáctica general, como tampoco con didáctica especial. Es plausible considerar que la neurodidáctica es una modalidad de las didácticas especiales, pero esto no la convierte en paradigma.

La neurodidáctica no tiene ninguna capacidad de predicción, como tampoco cuenta con una base epistemológica para sustentar sus criterios. En la misma dirección, no cuenta con una metodología para producir conocimiento neurodidáctico. Un factor positivo es que introduce una eventual explicación para potenciar el aprendizaje, al punto de asociarlo con subsanar una anomalia. No obstante, la anomalía la subsanan otras disciplinas, como la neurobiologia, por lo que no es parte de su propuesta epistemológica.

Sin embargo, la neurodidáctica no tiene por qué convertirse en un paradigma para demostrar su utilidad, menos su validez. Sin duda alguna, estamos ante una nueva especialidad profesional que responde directamente a los desafios de la educación del Siglo XXI. La neurodidáctica se puede asumir como una especialidad de estudio que incorpora los avances científicos sobre el funcionamiento del cerebro y el aprendizaje y la fundamentación teórica de la didáctica especial. Eso le permite diseñar propuestas de intervención educativa.

\section{Conclusiones}

En términos generales, el trabajo nos ha invitado a reflexionar sobre la importancia de la neurodidáctica como una especialidad de estudio en 
los procesos de enseñanza y aprendizaje. A continuación, se presentan en sintesis algunas conclusiones que se pueden derivar de este artículo de reflexión.

Siempre es atrevido hablar de un nuevo paradigma si se toma en cuenta la rigurosidad epistemológica del concepto paradigma. Los paradigmas se adoptan, la experiencia misma nos obliga a adoptarlos. Aunque también existe resistencia a incorporar paradigmas.

Un paradigma es un modelo teórico bien sustentado que permite explicar fenómenos de la realidad física, y desde luego, fenómenos sociales. En las ciencias sociales existen paradigmas. No obstante, por ser tan diversos los enfoques, son muchos los paradigmas. En educación podriamos considerar lo mismo: diversos enfoques educativos permiten la existencia de diferentes paradigmas para explicar el fenómeno que estudian.

Existe diferentes enfoques neurocientíficos que explican el proceso de aprendizaje. La neurobiología del aprendizaje se enfoca en sistematizar los diferentes estudios sobre el funcionamiento de las células del sistema nervioso y la forma en que el cerebro procesa la información que recibe del ambiente y la transforma en comportamientos observables.

Los modelos de enseñanza tradicionales, como el magistral, no toman en cuenta los estudios en el campo de las neurociencias relacionados con el cerebro y el aprendizaje. Esto dificulta la tarea del proceso de enseñanza y aprendizaje, en la medida en que no responde a diferentes variables, biológicas y ambientales, que influyen en un mejor aprendizaje en los estudiantes.

Pese a que la neurodidáctica es un campo de estudio enfocado a optimizar el aprendizaje con bases neurocientíficas, no se puede considerar un paradigma. 
La neurodidáctica no cumple con criterios conceptuales para considerarla un paradigma. Esta afirmación se hace en el contexto de la filosofía de la ciencia de Kuhn. No obstante, la neurodidáctica es una especialidad que permite diseñar propuestas de intervención para optimizar el aprendizaje, a partir de una sólida base neurocientífica y educativa tradicional.

La neurodidáctica supone la incorporación de una variedad de conocimientos provenientes de las neurociencias. El desconocimiento sobre la forma en que aprende el cerebro dificulta la tarea de los procesos de enseñanza y aprendizaje. Por el contario, una mayor amplitud de conocimientos sobre los procesos neurobiológicos del aprendizaje y de las características propias del cerebro, facilitan la capacidad de intervenir estratégicamente en el proceso de enseñanza y aprendizaje. A este conocimiento se le denomina neurodidáctica.

Finalmente, algunas investigaciones que se podrian derivar de este artículo de reflexión se relacionan con las bases epistemológicas y metodológicas de la neurodidáctica. Se podría hacer una investigación que exponga los criterios epistemológicos para considerar o no, a la neurodidáctica como un paradigma y, por otra parte, qué metodología implementa la neurodidáctica para producir conocimiento. 


\section{Referencias}

Adrover, D., Muñoz, E., Sánchez, I., \& Miranda, R. (2017). Neurobiología de los sistemas de aprendizaje y memoria. En D. R. Ripoll, Neurociencia cognitiva (págs. 411-438). Madrid: Panamericana.

Blaschke, J. (2014). Cerebro 2.0. . Barcelona: Robinbook.

Buxarrais, , M., \& Martínez, , M. (2015). Retos educativos para el siglo XXI: autonomía, responsabilidad, neurociencia y aprendizaje. Barcelona: Ediciones Octaedro .

Casasola Rivera, W. (2010). Una aproximación a la noción de paradigma. InformaTEC, 304.

Forés Miravalles, M., \& Ligioiz Vázquez, M. (2015). Descubrir la Neurodidáctica: aprender desde, en y para la vida. Cataluña: AOC.

Inostroza, F. (2018). Neuroe ducación social: hacia una pedagogia emocionalmente inclusiva. Chile: Ril editores.

Kuhn, T. (2004). La estructura de las revoluciones científicas. México: Fondo de Cultura Económica.

Landivar, A. (2012). Neuroeducación: educación para jóvenes bajo la lupa de María Montessori. Córdoba: Brujas.

Mora, F. (2016). Cuando el cere bro juega con las ideas. Madrid: Alianza Editorial.

Navarro Macías, R. (5 de Marzo de 2020). ¡Fuera neuromitos! Ni el cableado cerebral se completa a los tres años ni sobreestimular al niño le hace más listo. La Vanguardia. Recuperado el 1 de junio de 2020, de https:/ / www.lavanguardia.com/vivo/mamas-ypapas/20200305/473676833247/mitos-cerebro-ninos.html

Ortiz, T. (2015). Neurociencia y educación. Madrid: Alianza editorial.

Pérez, M. (2014). Cerebro que aprende: cómo apasionarnos con el conocimiento para transformar nuestra vida. Buenos Aires: Autoria Sherpa.

Pherez, G., Vargas, S., \& Jerez, J. (2018). Neuroaprendizaje, una propuesta educativa: herramientas para mejorar la praxis del docente. Civilizar Ciencias Sociales y Humanas, 18(34), 149-166. doi: https://doi.org/10.22518/usergioa/jour/ccsh/2018.1/a10

Portellano, J. A. (2005). Introducción a la neuropsicología. España: Madrid: McGraw-Hill.

Portellano, J., \& García, J. (2014). Neuropsicología de la atención, las funciones ejecutivas y la memoria. Madrid: SÍNTESIS.

Rodríguez de Ávila, U. (2020). ¡15 minutos de clase es suficiente! Psicobiología, Electrofisiología y Neuroeducación de la Atención Sostenida. Santa Marta: Ediciones Unimagdalena.

Uchite1, O. (2007). El lenguaje de las neuronas. Buenos Aires: Eudeba.

Ugaz Cayao, S., Fernández Cosavalente, H., Ugaz Cachay, L., Vásquez Vásquez, F., \& Quiroz Aldave, E. (2019). La neurobiologia aplicada: Bases del neurode sarrollo y aprendizaje. SCIÉNDO, 22(1), 169-173. doi: https://doi.org/10.17268/sciendo.2019.022 